\title{
MedienPädagogik
}

Zeitschrift für Theorie und Praxis der Medienbildung

Jahrbuch Medienpädagogik 4.

Zweitveröffentlichung aus: Jahrbuch Medienpädagogik 4. (2005) Wiesbaden: VS Verlag für Sozialwissenschaften. Hrsg. v. Ben Bachmair, Peter Diepold und Claudia de Witt.

\section{Neue Medien unter der Organisationsperspektive Eine empirische Untersuchung in der Weiterbildung}

\author{
Richard Stang
}

\section{Ausgangssituation}

Gesellschaftliche Modernisierungsprozesse haben zu immensen Veränderungsprozessen auf allen Ebenen gesellschaftlichen Lebens geführt. Wirtschaft, Politik, Bildungswesen etc. sind als Gesamtsysteme davon betroffen, genauso wie die Institutionen und Organisationen, die in deren Rahmen agieren. Stehr (2000, S. 17) spricht davon, dass wir uns in einem „Übergangsstadium zwischen zwei Gesellschaftsformationen“ befinden und meint damit den Übergang von der „Industriegesellschaft“ zur „Wissensgesellschaft“, in der Wissen konstitutiv für die Gesellschaftsformation ist. Mit dem Bedeutungszuwachs der Ressource „Wissen“ gehen Prozesse der voranschreitenden gesellschaftlichen Ausdifferenzierung einher.

So unterschiedlich die sozialwissenschaftlichen Analysen der Gesellschaftsformation und die daraus gefolgerten theoretischen Konstrukte auch sein mögen (vgl. u.a. Beck 1986, Castells 2001, Gross 1994, Schulze 1993), wird doch in einem besonderen Maße die Entwicklung von Technik, besonders der Informations- und Kommunikationstechniken (im Folgenden: Neuen Medien ${ }^{1}$ ), als ein wichtiger Motor der gesellschaftlichen Veränderungsprozesse gesehen. Besonders Castells (2001) hat die gesellschaftliche, kulturelle und ökonomische Bedeutung der Neuen Medien in seiner Studie über die Netzwerkgesellschaft eindrucksvoll herausgearbeitet.

Auch die Weiterbildung ist von diesen Veränderungsprozessen betroffen. Es erstaunt also nicht, dass in den letzten Jahren auch in der Weiterbildung die Auseinandersetzung mit den Neuen Medien zugenommen hat. Dabei ging es vor allem um neue Angebotskonzepte in diesem Bereich. E-Learning, Online-Lernen, computergestütztes Selbstlernen usw. sind Themenfelder, die hier die Diskussion sowohl in der Praxis als auch in der Forschung bestimmen. Interessant ist in diesem Zusammenhang, dass die Frage nach der Bedeutung der Neuen Medien für die institutionelle Organisation von Weiterbildung - sowohl im wissenschaftlichen Diskurs als auch in den Auseinan-

1 Der hier verwendete Begriff „Neue Medien“ umfasst alle computergestützten Techniken und Anwendungen, die u.a. im Weiterbildungsbereich eingesetzt werden, d.h. sowohl Hardware (Computer, Scanner, Digitale Kamera usw.), Software (Lernsoftware, Programmsoftware usw.) als auch computergestützte Netzwerke (z.B. Internet, Intranet). 
dersetzungen der Praxis - wenn überhaupt nur unter der Perspektive der Virtualisierung von Bildung in Form von Telelearning-Angeboten eine Rolle spielt. Die Frage, welche Auswirkungen die Neuen Medien auf die organisationale Struktur von traditionellen Bildungsinstitutionen haben, ist bisher von der Erwachsenenbildungsforschung, der Organisationsforschung und der medienpädagogischen Forschung nicht in den Blick genommen worden. Dies ist besonders vor dem Hintergrund bemerkenswert, dass die organisationale Struktur die Rahmung für das pädagogische Handeln darstellt.

Mit der hier vorgestellten Untersuchung soll eine Bestandsaufnahme im Bereich der Weiterbildung mit dem Fokus auf den Volkshochschulen und damit ein Beitrag zur Organisationsforschung in der Weiterbildung geleistet werden. Außerdem soll aufgezeigt werden, welche Bedeutung die Frage nach der Organisation von Bildungseinrichtungen auch für die (medien-)pädagogische Theoriedebatte hat. Nicht im Fokus stehen Fragen, wie sich didaktisch-methodische Konzeptionen und Lernarrangements durch die Neuen Medien verändern.

\section{Fragestellungen und Forschungsansatz}

Die gesellschaftlichen und wirtschaftlichen Veränderungen - z.B. Globalisierung, Informatisierung und Ökonomisierung - haben in den letzten Jahren dazu geführt, dass immer mehr Firmen und Institutionen gezwungen waren, ihre Organisation zu restrukturieren und den neuen Gegebenheiten anzupassen. Auch im Bereich der Weiterbildung ist seit Mitte der 1990er Jahre eine intensive Diskussion über die Veränderung der Organisationen und Organisationsentwicklung (OE) begonnen worden (vgl. Schäffter 2001). Auffallend bei den Diskussionen und bei den vielfältig in Angriff genommenen OE-Prozessen ist, dass das Thema „Neue Medien“ als Bestandteil der Veränderung von Organisationen keine explizite Berücksichtung fand und findet. Dies erstaunt umso mehr, als die Neuen Medien zu einer großen Veränderung von Arbeitsorganisation auf der einen Seite (z.B. Online-Anmeldungen) und von Angebotsstrukturen in der Weiterbildung auf der anderen Seite (z.B. computergestützte Selbstlernzentren) geführt haben.

Das Deutsche Institut für Erwachsenenbildung (DIE) hat mit dem Projekt „Mediale Innovationen und deren Auswirkungen auf die Organisation von Weiterbildung (MIA)“ den Fokus auf die Fragestellung gerichtet, wie sich die Neuen Medien auf die Organisation von Weiterbildungsinstitutionen auswirken. Da es im Bereich der Weiterbildung keine Untersuchungen gab, die sich explizit mit diesem Thema beschäftigten, stand zunächst die Frage nach dem „state of the art“ des Einsatzes und der Bedeutung Neuer Medien in Institutionen der Weiterbildung im Zentrum der Forschungsarbeit. Zentrale Fragestellungen dabei waren unter anderem: 
- Wie werden die Neuen Medien in Weiterbildungseinrichtungen genutzt?

- Welche Auswirkungen hat der Einsatz der Neuen Medien auf die Organisation der Einrichtungen?

- Welche Faktoren bestimmen den Umgang mit den Neuen Medien in Weiterbildungseinrichtungen?

- Wie könnten Ansätze für eine medienorientierte Organisationsentwicklung gestaltet sein?

Betrachten wir die drei Themenbereiche, auf die sich die vorliegende Untersuchung im Wesentlichen bezieht - Neue Medien, Organisation und Weiterbildung -, erweisen sich unterschiedliche Forschungsstränge und theoretische Zugänge als relevant für den theoretischen Bezugsrahmen. Deshalb bezieht sich der Forschungsansatz bei der vorliegenden Untersuchung auf Ansätze der Organisationsforschung, der medien- und technikbezogenen Forschung, der Erwachsenenbildungsforschung und nicht zuletzt der Innovationsforschung. Da sich der Forschungsstand bezogen auf das Themenfeld „Neue Medien und Organisation“ nicht durch eine einheitliche Theorie und empirische Forschungspraxis auszeichnet, wurde für die vorliegende Untersuchung ein interdisziplinärer Ansatz gewählt, der auf die unterschiedlichen Bezüge rekurriert, sich aber im Wesentlichen auf eine systemtheoretische Perspektive bezieht. Vor allem Luhmann (2000) liefert mit den Analysedimensionen „Kommunikation“, „Entscheidung“, „Reform“ und „Organisationskultur“ eine theoretische Rahmung für die Analyse.

Da eine Untersuchung des gesamten Weiterbildungsbereichs den Rahmen der finanziellen und personellen Ressourcen gesprengt hätte, konzentriert sich die Forschungsarbeit auf die Volkshochschulen als größten Träger der Weiterbildung in Deutschland. Nicht nur die herausragende Stellung der Volkshochschulen war ein Grund für diese Wahl, sondern auch die Tatsache, dass mit der Volkshochschul-Statistik die umfangreichste Weiterbildungsstatistik für einen einzelnen Träger vorliegt (vgl. Pehl/Reitz 2000), auf deren Daten im Rahmen des Forschungsprojekts zurückgegriffen werden konnte.

\section{Methodisches Vorgehen}

\section{$3.1 \quad$ Überblick}

Da es in der vorliegenden Forschungsarbeit darum geht, einen Themenkomplex empirisch zu erschließen, sind sowohl die auf quantitativen Methoden als auch auf qualitativen Methoden aufbauenden Zugänge eher als „entdeckende“ zu qualifizieren. Methodisch wird dabei die Verschränkung (Triangulation) der unterschiedlich gewonnenen Daten in den Mittelpunkt gestellt.

Als Ausgangspunkt diente eine schriftliche Befragung mit Hilfe eines standardisierten Fragebogens, der an alle 998 deutschen Volkshochschulen verschickt wurde. Der Rücklauf wurde mit Hilfe von deskriptiven und multi- 
variaten Analysemethoden ausgewertet. Diese Auswertung bildete die Grundlage für die Entwicklung eines Interviewleitfadens und für die Auswahl von Einrichtungen, in denen unter Verwendung der qualitativen Forschungsmethode „Experteninterview“ Interviews mit den Leitern und den EDV-Verantwortlichen geführt wurden.

Dabei sollte mit Hilfe der schriftlichen Befragung der aktuelle Status des Einsatzes Neuer Medien in den Einrichtungen erkundet werden, während die Experteninterviews dazu dienten, die Situation in konkreten Einrichtungen zu untersuchen, die sich in der Analyse der schriftlichen Befragung als medienorientiert herausgestellt haben.

\subsection{Schriftliche Befragung}

Als Befragungstechnik wurde eine schriftliche Erhebung in Form eines Fragebogens gewählt, da nur so die Berücksichtigung aller Volkshochschulen (998) bei der Befragung gewährleistet werden konnte. Um die 53 Fragen übersichtlicher und thematisch geordnet zu präsentieren, wurde der Fragebogen in die folgenden sechs thematischen Fragekomplexe aufgeteilt:

- Neue Medien in der Einrichtung

- Programmangebot der Einrichtung

- Neue Medien im Kontext der Organisation

- Neue Medien in ihrer Bedeutung für die Organisationsentwicklung

- Allgemeine Einschätzungen

- Zur Person

Die Daten, die über die Volkshochschulstatistik zur Verfügung standen (Siedlungsstruktur, Leitungsstruktur, Rechtsträgerschaft, Anzahl der hauptberuflichen Pädagog/innen, Kursangebote usw.), wurden nicht abgefragt, da diese über eine Codierung der Fragebogen zugeordnet werden konnten.

Im Rahmen eines Kooperationsvertrags mit dem Lehrstuhl für Konsum und Kommunikationsforschung an der Universität Trier (Prof. Dr. Michael Jäckel) wurde die Entwicklung des Fragebogens beraten, das Layout gestaltet sowie die Dateneingabe realisiert.

Um einen möglichst guten Überblick über die Situation an den Volkshochschulen zu bekommen, wurden alle deutschen Volkshochschulen (insgesamt 998) bei der Erhebung berücksichtigt. Dazu wurde die der Volkshochschul-Statistik zugrunde liegende Adressendatei genutzt. Insgesamt gingen bis zur Deadline 378 Fragebogen (37,9\%) ein. In Anbetracht des umfangreichen Fragebogens (53 Fragen auf 16 Seiten) ist dieser Rücklauf als sehr gut zu bezeichnen. 


\subsubsection{Analyse des Rücklaufs}

Unter folgenden Aspekten wurde ein Abgleich der Daten des Rücklaufs mit der Volkshochschulstatistik vorgenommen:

- Regionale Strukturierung

- Verteilung Alte/Neue Bundesländer

- Leitungsstruktur

- festangestellte hauptberuflich pädagogische Mitarbeiter/innen

- Rechtsträger

- Anzahl der IuK-Kurse.

Betrachtet man die sechs in die Analyse einbezogenen Strukturierungselemente in der Gesamtheit, wird deutlich², dass in der Tendenz eine große Übereinstimmung zwischen Rücklauf und Grundgesamtheit besteht. Doch fallen auch einige Abweichungen auf: Es sind vor allem die ehrenamtlich geleiteten Volkshochschulen, die Einrichtungen, die keine hauptberuflichen pädagogischen Mitarbeiter/innen fest angestellt haben und die, die weniger (unter 50) bzw. keine IuK-Kurse anbieten, die weniger vertreten sind, als es ihr Anteil an der Grundgesamtheit vermuten ließe. Ein Grund dafür könnte sein, dass vor allem „kleinere“ Einrichtungen nicht über die personellen Kapazitäten verfügen, sich an einer solchen Befragung zu beteiligen. Auch wenn der Rücklauf nicht auf jeder Ebene repräsentativ für die Grundgesamtheit gewertet werden kann, lässt die tendenzielle Übereinstimmung mit der Grundgesamtheit doch Rückschlüsse auf den Stand des Einsatzes Neuer Medien in Volkshochschulen in der Breite zu.

\subsection{Experten-Interviews}

Nach einer ersten deskriptiven Auswertung der schriftlichen Befragung ging es darum, Einrichtungen herauszufiltern, die für eine weiterführende Untersuchung bzgl. der Faktoren, die bedeutend für einen intensiven Einsatz Neuer Medien in den Institutionen sind, von besonderem Interesse sind, und in denen die Experten-Interviews (vgl. Meuser/Nagel 1997) durchgeführt werden können. Durch diesen Zugang lag es auf der Hand, dass eine Zufallsstichprobe aus dem gesamten Rücklauf hier nur bedingt weiterführen konnte, vor allem auch, weil aus forschungsökonomischen Gesichtspunkten nur eine begrenzte Anzahl von Einrichtungen einbezogen werden konnte (vgl. Lamnek 1993, S. 92ff.).

Deshalb wurde zunächst ein Weg gesucht, den Rücklauf stärker zu strukturieren und Gruppen zu bilden, die spezifische Einrichtungsprofile

2 Die detaillierte Darstellung der Analyse des Rücklaufs würde den Rahmen dieses Beitrages sprengen. Wer Interesse an der Analyse hat, kann diese bei dem Autor unter: stang@die-bonn.de nachfragen. 
bzgl. des Einsatzes Neuer Medien in der Einrichtung repräsentieren. Dies wurde mit Hilfe einer Clusteranalyse (vgl. dazu ausführlich: Bachhaus et al. 2000, S. 328ff.; Voß 2000, S. 287ff.) realisiert.

Bei der Clusteranalyse handelt es sich um ein Klassifikationsverfahren (vgl. Meiser/ Humburg 1996, S. 279). Die Zielsetzung ist die Zusammenfassung einer Vielzahl von Personen bzw. Objekten zu Gruppen, bei denen zwischen den einzelnen Mitgliedern der jeweiligen Gruppen eine sehr ähnliche Eigenschaftsstruktur vorliegt, zwischen den Gruppen selbst aber möglichst wenige Ähnlichkeiten vorliegen. Nach Backhaus et al. (2000, S. 329) geht es bei der Clusteranalyse darum, aus einer heterogenen Gesamtheit von Objekten - im vorliegenden Fall: Volkshochschulen - homogene Teilmengen von Objekten zu identifizieren.

Eine der wichtigsten Aufgaben bei der Clusteranalyse ist die Festlegung von Merkmalen, die die Basis für die Gruppenbildung liefern sollen. Der Weg, der in der vorliegenden Untersuchung gewählt wurde, war, auf der Grundlage der Auswertung der schriftlichen Befragung und der Daten aus der Volkshochschulstatistik bezogen auf den Rücklauf Merkmale herauszufiltern, die sich sehr unterschiedlich im Rücklauf ausprägten. Da die Clusteranalyse im vorliegenden Fall nicht dazu diente, eine umfassende Typologie von Einrichtungen bezogen auf den Einsatz Neuer Medien zu entwickeln, sondern als Grundlage für die Auswahl der Einrichtungen, in denen Expert/innen-Interviews durchgeführt werden sollten, wurde davon abgesehen, alle vorliegenden Merkmale aus der Befragung und der Volkshochschulstatistik in die Clusteranalyse mit einzubeziehen.

Folgende Aspekte bezogen auf die Institutionen wurden in den Blick genommen:

- technische Ausstattung bezogen auf Kommunikation (intern/extern),

- technische Ausstattung bezogen auf die Durchführung des Angebots,

- inhaltliches Angebot im Bereich Neue Medien,

- personale Struktur im Bezug auf Neue Medien und

- die Vernetzung nach außen im Kontext des Angebots im Bereich Neue Medien.

Auf Basis dieser thematischen Konturierung wurden folgende Merkmale für die Clusteranalyse ausgewählt und auf binäre Variablen reduziert:

- Intranet (vorhanden $=1 /$ nicht vorhanden $=0$ ),

- Homepage (vorhanden $=1 /$ nicht vorhanden $=0$ ),

- eigene Computerräume (vorhanden $=1 /$ nicht vorhanden $=0$ ),

- Kurse im Bereich Informations- und Kommunikationstechniken (bis 50 Kurse = 0/ über 50 Kurse $=1$ ),

- hauptberuflicher EDV-Organisator (vorhanden = 1/nicht vorhanden =0),

- Auftragsmaßnahmen im Bereich „Neue Medien“ $(\mathrm{Ja}=1 /$ Nein $=0)$. 
Unter Verwendung von SPSS wurde eine Gruppenbildung mit Hilfe der „Clusterzentrenanalyse“ durchgeführt (vgl. Voß 2000, S. 296ff.). Diese führte zu folgenden Clustern:

\begin{tabular}{llccr}
\hline & Cluster 1 & Cluster 2 & Cluster 3 & Cluster 4 \\
\hline Intranet & 1 & 1 & 1 & 0 \\
Homepage & 1 & 1 & 1 & 0 \\
Eigene Computerräume & 1 & 1 & 1 & 0 \\
IuK-Kurse & 1 & 1 & 1 & 0 \\
EDV-Organisator & 1 & 0 & 0 & 0 \\
Auftragsmaßnahmen & 1 & 0 & 1 & 0 \\
\hline
\end{tabular}

Tab. 1: Clusterzentren.

68 Einrichtungen wurden Cluster 1 zugeordnet, 67 Cluster 2, 141 Cluster 3 und 102 Cluster 4. Betrachtet man sich diese vier Cluster wird deutlich, dass es drei Clusterzentren gibt, die sich nur in einem bzw. zwei Merkmalen unterscheiden, während das vierte Clusterzentrum sich gravierend von den anderen unterscheidet. Wenn wir die Cluster bezogen auf die Struktur des Einsatzes Neue Medien in den Einrichtungen betrachten heißt das, dass in den Einrichtungen von Cluster 1-3 der Einsatz Neuer Medien eine höhere Bedeutung hat als in denen von Cluster 4. Interessant ist in diesem Zusammenhang die Frage, welche weiteren Faktoren die Einrichtungen kennzeichnen, die sich in den jeweiligen Clustern finden. Um die einzelnen Cluster dahingehend zu untersuchen und die Auswahl der Einrichtungen für die Experteninterviews voranzutreiben, wurden diese unter Zuhilfenahme weiterer Merkmale analysiert.

Durch die Analyse bivariater Verteilungen (vgl. Voß 2000, S. 91ff.) zwischen den Clustern und ausgewählten Merkmalen entstand ein detaillierteres Bild der Struktur der Einrichtungen innerhalb der jeweiligen Cluster. Die bivariate Analyse wurde anhand folgender Merkmale durchgeführt, die sich unterschiedlichen Strukturbereichen zuordnen lassen:

Siedlungs- und Bevölkerungsstruktur

- Bundesland

- Regionalstruktur

- Bevölkerungsdichte

- Einwohnerstruktur

Institutionsstruktur

- Rechtsträger

- Leitungsstruktur

- Struktur hauptberufliche Pädagog/innen

- Status der Organisationsentwicklung 
Struktur im Bezug auf Neue Medien

- Rolle der Neuen Medien in der Organisationsentwicklung

- besondere Angebote (Telelearning, Internet Cafe, Selbstlernzentrum)

Bündelt man nun die einzelnen Ergebnisse der Analyse der Cluster, wählt einige besonders prägnante Ergebnisse aus und betrachtet man diese in ihren extremen Ausprägungen, ergibt sich zusammenfassend folgendes Bild für die vier Cluster³

\begin{tabular}{|c|c|c|c|c|}
\hline & Cluster 1 & Cluster 2 & Cluster 3 & Cluster 4 \\
\hline Intranet & vorhanden & vorhanden & vorhanden & nicht vorhanden \\
\hline Homepage & vorhanden & vorhanden & vorhanden & nicht vorhanden \\
\hline $\begin{array}{l}\text { Eigene Computer- } \\
\text { räume }\end{array}$ & vorhanden & vorhanden & vorhanden & nicht vorhanden \\
\hline \multirow[t]{2}{*}{ IuK-Kurse } & über & über & über & unter \\
\hline & 50 Kurse & 50 Kurse & 50 Kurse & 50 Kurse \\
\hline EDV-Organisator & vorhanden & nicht vorhanden & nicht vorhanden & nicht vorhanden \\
\hline Auftragsmaßnahmen & gibt es & gibt es nicht & gibt es & gibt es nicht \\
\hline Einwohnerzahl & über 50.000 & über $25.000^{4}$ & über $25.000^{5}$ & bis 25.000 \\
\hline Leitungsstruktur & hauptberuflich & hauptberuflich & hauptberuflich & ehrenamtlich \\
\hline Anzahl HPM & Über 4 HPM & $1-10 \mathrm{HPM}^{6}$ & 1,1-10 НРМ & bis $1 \mathrm{HPM}$ \\
\hline $\begin{array}{l}\text { Besondere medienbe- } \\
\text { zogene Angebote }\end{array}$ & vorhanden & nicht vorhanden & vorhanden & nicht vorhanden \\
\hline
\end{tabular}

Tab. 2: Struktur der Cluster.

Da es bei den Experteninterviews vor allem darum gehen sollte, im Bezug auf den Einsatz Neuer Medien besonders interessante Einrichtungen in den Blick zu nehmen, wurde nach der Analyse der Cluster entschieden, Volkshochschulen aus dem Cluster 4 nicht zu berücksichtigen. Hier lassen sich besonders viele Volkshochschulen finden, die unter anderem:

- einen Einzugsbereich von unter 25.000 Einwohnern haben (50\%),

- ehrenamtlich geführt sind (43,1\%),

- keine HPM haben (66,7\%).

Dass es sich in Cluster 4 vor allem um „kleine“ Einrichtungen mit unter anderem geringer personeller Ausstattung und einem kleinen Einzugsgebiet handelt, ist sicher auch ein entscheidender Grund für die fehlende Ausstattung und den nicht ausgeprägten Einsatz im Bezug auf Neue Medien. Sicher wäre es in weiterführenden Forschungsarbeiten auch von Interesse, zu untersuchen, welche Faktoren in Einrichtungen eine Rolle spielen, dass die Entwicklung im Bereich der Neuen Medien nicht besonders vorangetrieben

3 Grundlage bilden hier Werte der Ausprägungen, die in der Tendenz höher vorhanden waren als erwartet.

4 Bei der Kategorie „100.001-250.000“ liegt hier der Wert unter dem erwarteten.

5 Bei der Kategorie „über 250.000“ liegt hier der Wert unter dem erwarteten.

6 Bei den Kategorien „1,1-2 HPM“, „4,1-5 HPM“ liegt hier der Wert unter dem erwarteten. 
wird. Doch aus forschungsökonomischen Gründen wurden in der vorliegenden Untersuchung besonders die „positiven“ Fälle in den Blick genommen.

Für die Auswahl aus den Clustern 1-3 wurden nach diesem Prinzip ,extreme Fälle’ und die ,Auswahl nach dem Konzentrationsprinzip’ gewählt (vgl. Schnell et al. 1995, S. 279ff.). Dazu wurden auf den einzelnen Ebenen der Analyse der Cluster besonders auffällige und besonders typische Einrichtungen anhand der Codierungsnummer herausgefiltert, z.B. ehrenamtlich geführte Einrichtungen, die sich in Cluster 1 finden. Einrichtungen, die auf unterschiedlichen Ebenen herausgefiltert wurden, wurden nochmals besonders hervorgehoben. Diese wurden dann nach strukturellen Daten wie Regionaltypen, Bundesland, Rechtsform, Anzahl der HPM und Anzahl der IuK-Kurse abgeglichen. Als entscheidendes Kriterium bei der Konzentration wurden dann die besonderen medienbezogenen Angebote herangezogen.

\begin{tabular}{|c|c|c|c|c|c|}
\hline & \multicolumn{5}{|c|}{ Einrichtung } \\
\hline & A & B & $\mathrm{C}$ & $\mathrm{D}$ & $\mathrm{E}$ \\
\hline Cluster & 2 & 2 & 1 & 3 & 1 \\
\hline Bundesland & neues & altes & altes & altes & altes \\
\hline Regionaltyp & $\begin{array}{l}\text { AR mit } \\
\text { heraus-rag. } \\
\text { Zentrum }\end{array}$ & $\begin{array}{l}\text { LR geringere } \\
\text { Dichte }\end{array}$ & $\begin{array}{l}\text { LR höhere } \\
\text { Dichte }\end{array}$ & $\begin{array}{l}\text { Hochverdich- } \\
\text { teter AR }\end{array}$ & $\begin{array}{l}\text { AR mit } \\
\text { heraus-rag. } \\
\text { Zentrum }\end{array}$ \\
\hline Einwohnerzahl & ca. 100.000 & са. 130.000 & са. 90.000 & ca. 20.000 & са. 200.000 \\
\hline $\begin{array}{l}\text { Bevölkerungs- } \\
\text { dichte }\end{array}$ & über 50 & über 1000 & über 50 & über 500 & über 1500 \\
\hline $\begin{array}{l}\text { Rechtsträger } \\
\text { Leitungs- } \\
\text { struktur }\end{array}$ & $\begin{array}{l}\text { Gemeinde } \\
\text { hauptamtlich }\end{array}$ & $\begin{array}{l}\text { Kreis } \\
\text { hauptamtlich }\end{array}$ & $\begin{array}{l}\text { Kreis } \\
\text { ehrenamtlich }\end{array}$ & $\begin{array}{l}\text { e.V. } \\
\text { hauptamtlich }\end{array}$ & $\begin{array}{l}\text { Gemeinde } \\
\text { hauptamtlich }\end{array}$ \\
\hline Außenstellen & 0 & über 20 & über 20 & unter 5 & über 5 \\
\hline HPM & über 5 & 0 & 0 & über 5 & über 40 \\
\hline $\begin{array}{l}\text { Verwaltungsmit- } \\
\text { arbeiter }\end{array}$ & über 5 & unter 5 & unter 5 & unter 5 & über 30 \\
\hline Kursleiter & über 300 & über 150 & über 200 & über 90 & über 580 \\
\hline Kurse insgesamt & über 850 & über 450 & über 650 & über 400 & über 2000 \\
\hline IuK-Kurse & über 110 & über 130 & über 170 & über 110 & über 350 \\
\hline $\begin{array}{l}\text { Besondere me- } \\
\text { dienbez. Ange- } \\
\text { bote }\end{array}$ & $\begin{array}{l}\text { Selbstlern- } \\
\text { zentrum }\end{array}$ & $\begin{array}{l}\text { Telelearning, } \\
\text { Internet-Cafe, } \\
\text { Selbstlern- } \\
\text { zentrum }\end{array}$ & keine & $\begin{array}{l}\text { Selbstlern- } \\
\text { zentrum }\end{array}$ & Telelearning \\
\hline
\end{tabular}

Tab. 3: Die ausgewählten Einrichtungen.

Nach der Auswahl nach „extremen Fällen“ und dem Konzentrationsprinzip wurden auch unter forschungsökonomischen Gesichtspunkten - fünf Einrichtungen ausgewählt, wobei für jedes Cluster eine eher typische Einrichtung gewählt wurde und für Cluster 1 und 2 jeweils eine Einrichtung unter der Perspektive des „extremen Falls“. Für Cluster 1 bedeutete das, eine ehrenamtlich geführte Einrichtung zu nehmen, für das Cluster 2 wurde eine 
Einrichtung ohne HPM ausgewählt. Nimmt man nun die fünf Einrichtungen in den Blick, ergibt sich folgendes Bild bezogen auf ausgewählte Aspekte?:

Im Wechselspiel von Organisation und Neuen Medien kommt vor allem den Menschen, den Akteuren, eine besondere Bedeutung zu (vgl. Schwarzer/Krcmar 1999, S. 92). Das war der zentrale Grund dafür, den Blick auf einzelne Akteure als Expert/innen der ausgewählten Einrichtungen zu richten.

Die Auswahl erfolgt auf der Basis der Zuordnung von Sonderwissen (vgl. Schütz 1972), über das die interviewten Personen durch ihre Rollen in den jeweiligen Institutionen verfügen. Dabei geht es vor allem darum, Personen in den Blick zu nehmen, die bezogen auf die Gesamtinstitution und bezogen auf die Neuen Medien über ein Sonderwissen verfügen. Konkret bedeutet das, dass in den ausgewählten Einrichtungen jeweils mit dem Leiter und dem Verantwortlichen für die technische Betreuung der EDV Experteninterviews geführt wurden ${ }^{8}$. Der Leiter wurde gewählt, weil er die gesamte Organisation im Blick hat und die strategische Ausrichtung der Institution entscheidend bestimmt. Der Verantwortliche für die technische Betreuung der EDV wurde gewählt, weil er die technische Infrastruktur im Blick hat und strategische Entscheidungen der Einrichtung unter einer technikbezogenen Perspektive beurteilen kann.

Die Experten-Interviews wurden anhand eines Leitfadens, der sich an den zentralen Punkten der schriftlichen Befragung orientierte, Anfang 2002 in den jeweiligen Einrichtungen durchgeführt. Die Auswertung der Interviews erfolgte mit Hilfe der Software MAXqda.

\section{Ausgewählte Ergebnisse}

Die folgende Darstellung ausgewählter Ergebnisse bezieht sich auf einige zentrale Punkte der schriftlichen Befragung ergänzt durch Ergebnisse der Experteninterviews in ausgewählten Einrichtungen. Dabei werden folgende Themen in den Blick genommen:

- technische Infrastruktur

- Angebot

- Dienstleistung

- Kooperation und Konkurrenz

7 Die Codierung der Einrichtungen mit Buchstaben erfolgte nach Abfolge der Besuche zu den Experteninterviews in den Einrichtungen. Die verwendeten Daten stammen aus dem Datenbestand für die Volkshochschulstatistik für das Jahr 1999 (vgl. auch Pehl/Reitz 2000), da diese der Auswertung der schriftlichen Befragung zugrunde gelegt wurden. Einigen Angaben werden nur ungefähr gemacht, weil sonst die Einrichtungen über die genauen Daten zuzuordnen wären.

8 Die durch die Clusteranalyse und die weiterführende Analyse vorgenomme Auswahl der Institutionen führte dazu, dass die Interviewpartner alle Männer waren. 
- Neue Medien und Organisationsentwicklung

- Situation in ausgewählten Einrichtungen.

\subsection{Technische Infrastruktur}

92,5\% der Volkshochschulen verfügen Anfang 2001 über einen Internetzugang, 73,3\% der Einrichtungen sind mit einer eigenen Homepage im Internet vertreten. Da die Bedeutung des Internets seit Mitte der 1990er Jahre im gesellschaftlichen Diskurs zugenommen hatte und das Internet als eine der technischen Basisinnovationen angesehen werden kann, interessierte im Rahmen der Untersuchung nicht nur, ob ein Internetzugang bei den Institutionen vorhanden ist, sondern auch, wann dieser installiert wurde, um einen Blick auf die Innovationsprozesse zu werfen. Einen wichtigen theoretischen Bezugspunkt bei der Untersuchung dieser Frage bildeten die Arbeiten von Rogers (1995), der sich bereits Anfang der 1960er Jahre der Erforschung von Innovationsprozessen widmete. Rogers beschäftigte sich vor allem mit der Frage der Verbreitung („Diffusion“) von Innovationen. Diffusion definiert er „as the process by which an innovation is communicated through certain channels over time among the members of a social system“ (Hervorh. im Original; ebd., S. 10).

Betrachtet man nun den Zeitpunkt der Installation des ersten Internetzugangs in den Institutionen, ergibt sich folgendes Bild:

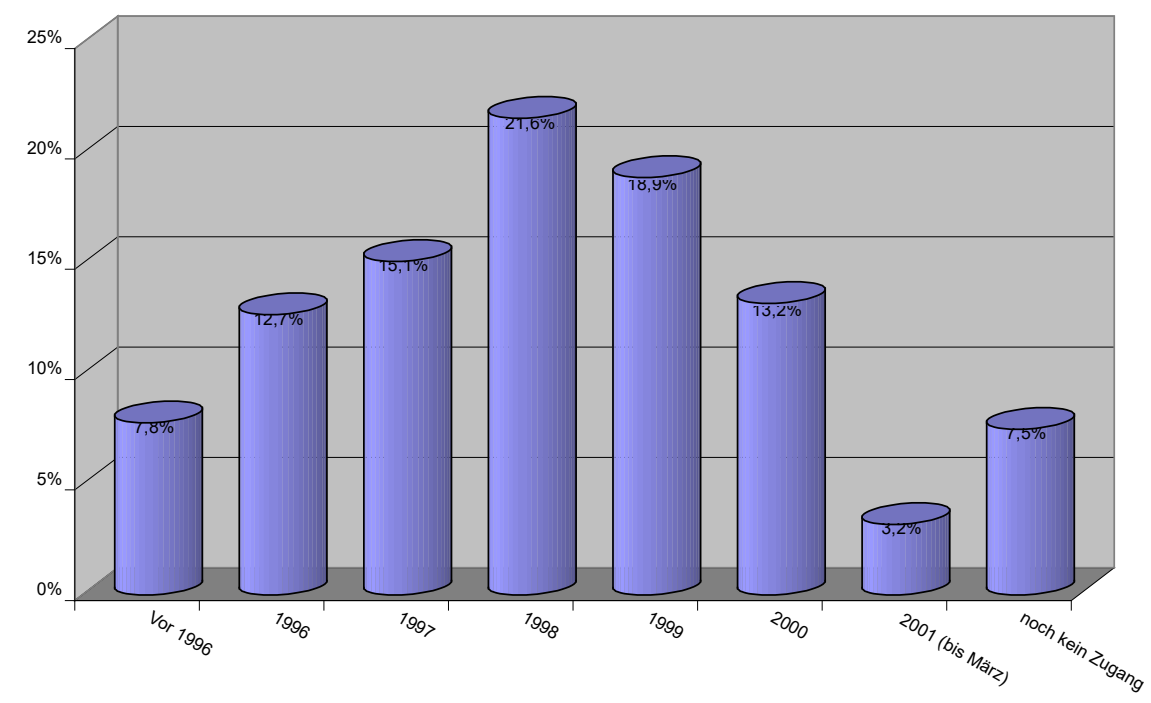

Abb. 1: Installation des ersten Internetzugangs ( $\mathrm{N}=371)$. 
Rogers (1995: 262) hat aufgrund seiner Untersuchungen folgende Benutzerkategorien in Relation zum Zeitpunkt der Adoption von Innovationen als Idealtypen entwickelt (vgl. Abb.2):

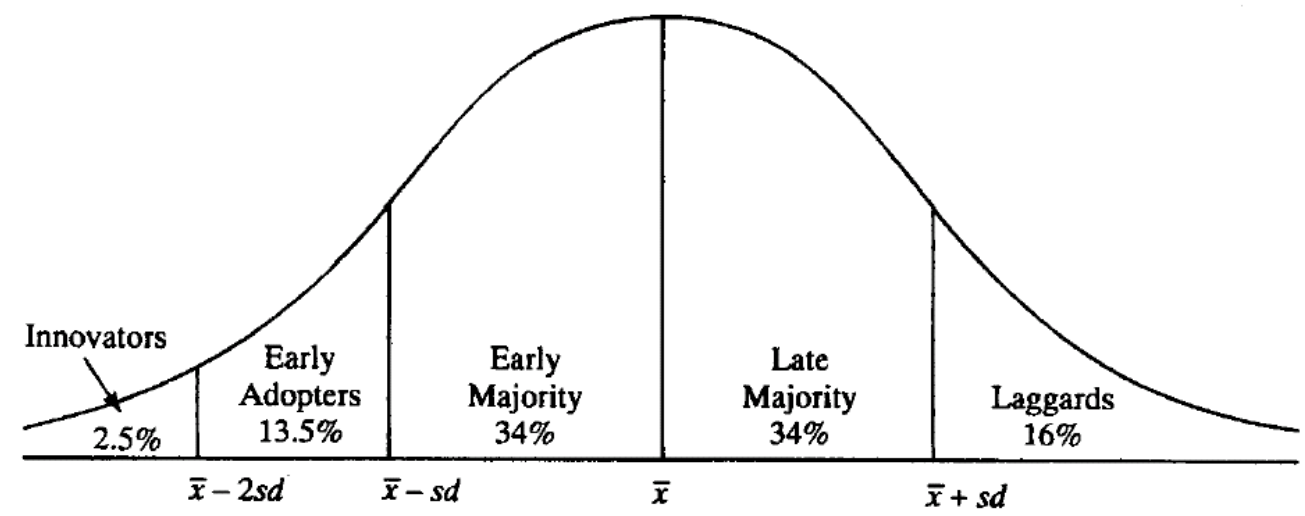

Abb. 2:

Nimmt man diese Strukturierung und vergleicht damit das Ergebnis der Erhebung, wird deutlich, dass man bezogen auf die Interneteinführung bei Volkshochschulen diese Kategorisierung übernehmen kann. Hier gibt es - vor allem wenn man den Wert für 2001 auf das gesamte Jahr hochrechnet - eine große Annäherung an die Verteilung der Idealtypen von Rogers. Das heißt, dass das Innovationsverhalten im System der Volkshochschulen bezogen auf den Faktor „Installation des ersten Internetzugangs“ als typisch für Innovationsprozesse bezeichnet werden kann. Interessant ist es, in weiterführenden Untersuchungen die Faktoren herauszuarbeiten, die für Zuordnung der Einrichtungen ausschlaggebend sind, d.h. welche Faktoren dazu beitragen, dass Institutionen Innovationen zu einem frühen Zeitpunkt adaptieren.

Neben Internetzugang und Homepage als Anwendungsdimensionen lässt sich die technische Infrastruktur unter anderem über den Versorgungsgrad der Mitarbeiter/innen mit Hardware und über die Ausstattung der Einrichtung mit Computerräumen beschreiben. Diese beiden Aspekte sind Indikatoren dafür, wie stark die Neuen Medien in der Organisation verankert sind.

Der Computer ist heute ein kaum mehr wegzudenkendes Arbeitsmittel, bezogen auf Bildungseinrichtungen sowohl für den Verwaltungsbereich als auch für den Bereich der pädagogischen Planung. Betrachtet man den Versorgungsgrad der festangestellten Mitarbeiter/innen in Volkshochschulen mit einem eigenen Computer an deren Arbeitsplatz ergibt sich folgendes Bild:

- Beim Verwaltungspersonal verfügen im Durchschnitt 97,24\% ( $N=351$; Standardabw.: 14,14) über einen eigenen Computer an ihrem Arbeits- 
platz, dabei ist in 94\% der Einrichtungen eine Vollversorgung mit 100\% gegeben.

- Beim pädagogischen Personal verfügen im Durchschnitt 91,08\% $(N=311 ; 25,73)$ über einen eigenen Computer an ihrem Arbeitsplatz, dabei ist in 85,5\% der Einrichtungen eine Vollversorgung mit 100\% gegeben.

Im Bereich der Verwaltung kann annähernd von einer Vollversorgung gesprochen werden, im pädagogischen Bereich ist diese noch nicht erreicht. Das deutet darauf hin, dass der Computer im Verwaltungsbereich von zentraler Bedeutung ist, während im Alltag des pädagogischen Bereiches die Notwendigkeit eines permanenten Zugriffs auf einen Computer anscheinend (noch) nicht gegeben ist.

79\% der Einrichtungen verfügen über eigene Computerräume für Bildungsangebote und Schulungen. Bei der Anzahl der Computerräume ergibt sich ein Mittelwert von 3,11 ( $N=290$; Standardabw. 5,10; Maximum: 64; Median: 2,00).

\begin{tabular}{ll}
\hline Ein Computerraum & $26,6 \%$ \\
Zwei Computerräume & $36,2 \%$ \\
Drei Computerräume & $14,8 \%$ \\
Vier Computerräume & $10,7 \%$ \\
Mehr als vier Computerräume & $11,7 \%$ \\
\hline
\end{tabular}

Tab. 4: Anzahl der Computerräume.

Drei Viertel der Einrichtungen, die über eigene Computerräume verfügen, nutzen zusätzlich Computerräume anderer Einrichtungen (75,5\%; $N=294)$. Die Mehrheit nutzt Computerräume von Schulen (69\%). 11,9\% nutzen die Räume von privaten Bildungsanbietern und 7,5\% von anderen öffentlich geförderten Weiterbildungseinrichtungen.

Die Nutzung der Computerräume von Schulen dominiert auch bei den Einrichtungen, die über keine eigenen Computerräume verfügen (87,3\%; $N=79)$. Computerräume von privaten Bildungsanbietern $(13,9 \%)$ und anderen öffentlich geförderten Weiterbildungsinstitutionen $(11,4 \%)$ werden deutlich weniger genutzt.

Die technische Infrastruktur in Volkshochschulen legt also nahe, dass hier die Voraussetzungen für die Entwicklung einer medienorientierten Organisation sehr gut sind. Wie die Neuen Medien allerdings in organisatorische Kontexte und die Entwicklung der Einrichtungen eingebunden sind, ist eine Frage, die sich eher beantworten lässt, wenn man andere Aspekte der Untersuchung in die Analyse mit einbezieht. 


\subsection{Angebot}

Das Angebot der Volkshochschulen konzentriert sich auch im Bereich Neue Medien (als Thema und Unterrichtsmittel ${ }^{9}$ ) traditionell auf Einzelveranstaltungen, Kurse (ohne Programmschulungen) und Programmschulungen (Schulungen für Anwenderprogramme wie Word, Excel usw.). Betrachtet man die Verteilung der Angebote bezogen auf die Anzahl der Veranstaltungen in den unterschiedlichen Programmbereichen für das Jahr 2000, wird deutlich, dass nach wie vor der überwiegende Teil der Angebote im Programmbereich „Arbeit - Beruf“ zu finden sind. In einer Untersuchung von 1998, die am Deutschen Institut für Erwachsenenbildung durchgeführt wurde und eine Stichprobe von Volkshochschulen mit ausschließlich hauptberuflicher Leitung als Grundlage hatte, waren 91,5\% ( $\mathrm{N}=101)$ der Angebote in diesem Programmbereich zu finden (vgl. Wagemann/Stang 1999: 117). Dass sich bei der vorliegenden Befragung der Wert noch erhöht hat, kann daran liegen, dass beim Rücklauf die Gesamtstruktur der Volkshochschulen berücksichtigt ist.

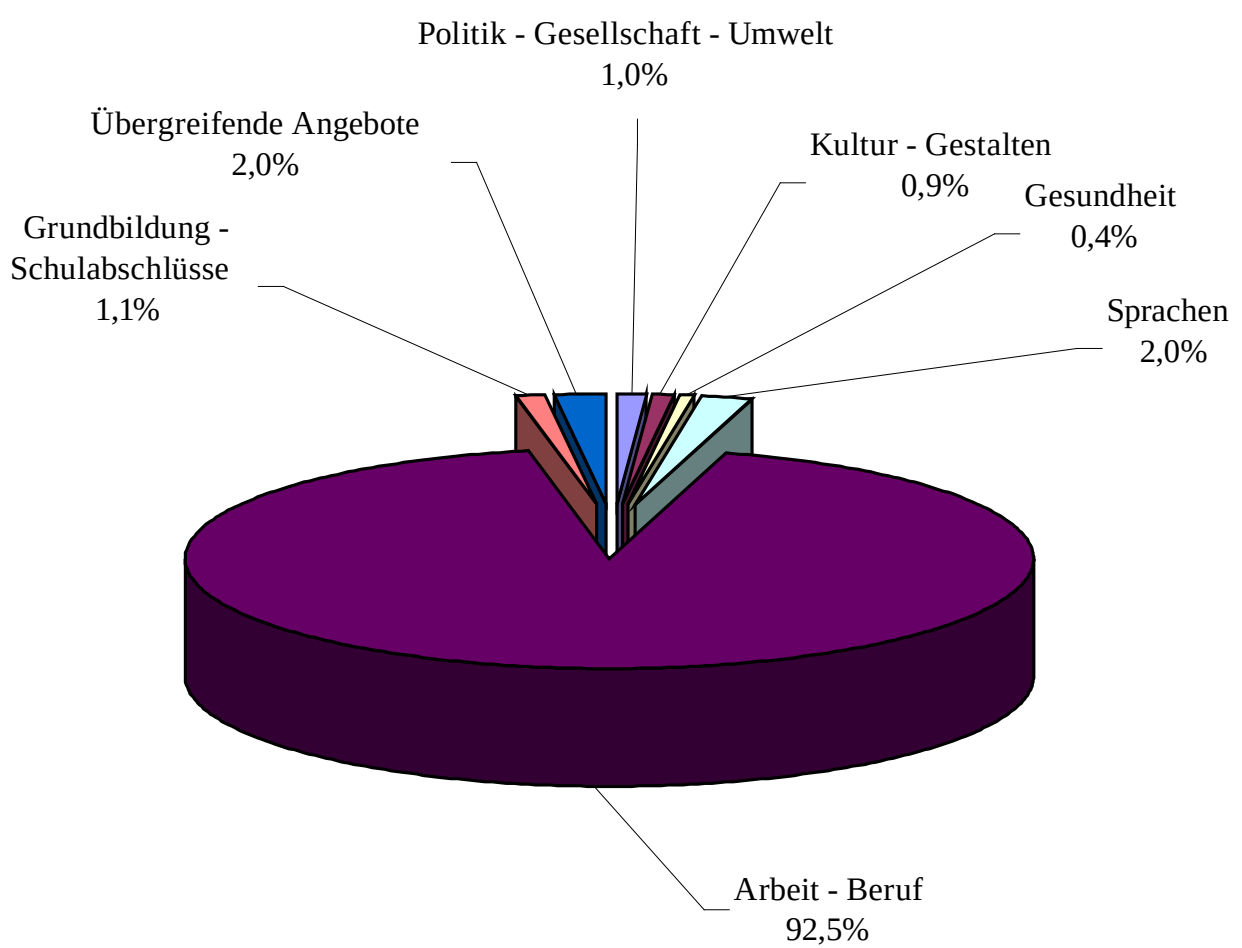

Abb. 3: Verteilung der Angebote auf die Programmbereiche $(\mathrm{N}=295)$.

9 Da die Analyse des Programmangebots nicht im Zentrum dieser Arbeit steht, wurden hier zur Reduzierung des Fragenkatalogs die Veranstaltungen, die Neue Medien zum Thema haben bzw. als Unterrichtsmittel einsetzen, gebündelt. Eine detaillierte Analyse des Programmangebots und des Einsatzes Neuer Medien in Veranstaltungen der Erwachsenenbildung ist ein weiteres Forschungsprojekt, das auf seine Bearbeitung wartet. 
Die Ausrichtung des Angebots auf den Programmbereich „Arbeit - Beruf“ macht ein Dilemma der Volkshochschulen deutlich. Um die technische Infrastruktur und die permanente Aktualisierung zu finanzieren, wird der Schwerpunkt auf nachgefragte Programmschulungen gelegt (Durchschnitt: 81,36 pro Einrichtung; Standardabw. 114,96; $\mathrm{N}=348$ ), Einzelveranstaltungen (Durchschnitt: 10,05 pro Einrichtung; Standardabw. 44,30; N = 292) und Kurse (ohne Programmschulungen) (Durchschnitt: 35,75 pro Einrichtung; Standardabw. 62,09; N = 292) spielen keine so große Rolle. Eine These, die in diesem Zusammenhang formuliert werden könnte, lautet: Dadurch, dass die technische Infrastruktur mit den Programmschulungen meistens ausgelastet ist, bleibt kaum Raum für Experimente in anderen Programmbereichen, in denen die Nachfrage nach Angeboten im Bereich Neue Medien erst entwickelt werden muss.

Neue, über das traditionelle Angebot der Einrichtungen hinausgehende medienbezogene Angebote gibt es bislang nur punktuell. Auf die Frage, welche anderen medienbezogenen Angebote es in den Einrichtungen gibt, antworteten 75,1\% ( $\mathrm{N}=353$ ) mit „Keine“. Über ein Internet-Café verfügen 11,9\% und über ein computergestütztes Selbstlernzentrum 7,1\%. 3,7\% machen ein Telelearning-Angebot ${ }^{10}$ und unter den 7,1\% „Sonstigen“ finden sich neben einem kostenlosen Beratungsangebot und einem Teleworking-Center vor allem spezielle Kurse und traditionelle Medienangebote wie Tonstudio und Bürgerfunk.

Die Tendenz, spezielle Angebote eher nicht im Blick zu haben, wenn es um Neue Medien geht, zeigt sich auch bei der Einschätzung, welche Rolle die Neuen Medien in den Einrichtungen im Rahmen spezieller Angebote (z.B. Internet-Café) spielen. ${ }^{11}$ Bündelt man die Skalenwerte „1“ (,überhaupt keine“) und „2“ („,keine“), dann sind das $50,4 \%(N=336)$.

Diesem Ergebnis in der Tendenz entgegengesetzt ist die Einschätzung zur Aussage „Der Einsatz Neuer Medien in der Weiterbildung bedarf eines verstärkten Experimentierens mit neuen Lehr-/Lernformen" ${ }^{“ 12}$. 49,1\% $(\mathrm{N}=369)$ stimmen dieser Aussage zu (,5“; 32,8\%) bzw. „voll und ganz zu“ (,6“; 16,3\%). Das deutet darauf hin, dass auf theoretischer Ebene die Notwendigkeit des Experiments gesehen wird, die praktische Umsetzung eher schwierig ist.

10 In einer Befragung zur „Bestandsaufnahme Telelernen in Volkshochschulen“ des Netzwerk Beruf und Weiterbildung des Deutschen Volkshochschul-Verbandes (DVV) vom Mai 2001 geben 9,3\% der befragten Institutionen ( $\mathrm{N}=162)$ an, Telelern-Angebote anzubieten (vgl. Rudolf 2001: 83). Bei dieser Untersuchung wurden allerdings nur 800 der 998 Volkshochschulen angeschrieben, was auf eine Vorselektion schließen lässt.

11 Die Einschätzung der Rolle konnte anhand einer Skala von 1 bis 6 vorgenommen werden (Skalenwert „1“ = „Überhaupt keine“; Skalenwert „, 6 “ = „Sehr große“).

12 Diese Aussage sollte anhand einer Skala von 1 bis 6 eingeschätzt werden (Skalenwert „1“ = „Stimme überhaupt nicht zu“; Skalenwert „,“ = „Stimme voll und ganz zu“). 


\subsection{Dienstleistung}

Besonders die Konzentration auf Programmschulungen hat Volkshochschulen in den letzten Jahren zu einem wichtigen Bildungsanbieter im Bereich Neue Medien werden lassen. Die Aktivitäten in diesem Bereich konzentrieren sich allerdings nicht nur auf die eigenen Angebote, die Einrichtungen sind auch zu Dienstleistern für andere Auftraggeber geworden. 60,3\% ( $\mathrm{N}$ = 375) der Einrichtungen führen Auftragsmaßnahmen im Bereich Neue Medien durch. Dabei sind die Auftraggeber folgende (Mehrfachnennungen waren möglich):

\begin{tabular}{ll}
\hline Firmen & $81,4 \%$ \\
Behörden & $69,0 \%$ \\
Arbeitsamt & $51,3 \%$ \\
Sonstige & $9,8 \%$ \\
\hline
\end{tabular}

Tab. 5: Auftraggeber von Maßnahmen im Bereich Neue Medien $(\mathrm{N}=226)$.

Unter den Sonstigen werden Einrichtungen wie z.B. Bundeswehr, Banken, Lehrerfortbildung und Vereine genannt. Rechnet man diese Zahlen auf die Einrichtungen, die sich an der Befragung beteiligt haben ( $\mathrm{N}=378$ ), hoch, dann übernehmen $49 \%$ der Einrichtungen Auftragsmaßnahmen für Firmen, 41,6\% für Behörden und 30,9\% für das Arbeitsamt.

\subsection{Kooperation und Konkurrenz}

Nicht nur die Dienstleistung für andere Institutionen und Firmen bildet die Vernetzung mit externen Einrichtungen ab, sondern auch die Zusammenarbeit mit anderen Institutionen im Bereich Neue Medien. 55,5\% ( $\mathrm{N}=375)$ arbeiten mit anderen Institutionen zusammen. Diese Kooperation geschieht auf den unterschiedlichsten Ebenen. Folgende Tabelle veranschaulicht das Netz und die Intensität der Kooperation (Mehrfachnennungen waren möglich).

\begin{tabular}{lllll}
\hline & $\begin{array}{l}\text { Gemeinsame } \\
\text { Angebote }\end{array}$ & $\begin{array}{l}\text { Gemeinsame } \\
\text { Nutzung von } \\
\text { Ressourcen }\end{array}$ & $\begin{array}{l}\text { Austausch von } \\
\text { Kursleitenden }\end{array}$ & Sonstiges \\
\hline $\begin{array}{l}\text { Private kommerzielle } \\
\begin{array}{l}\text { Bildungsanbieter } \\
\text { Öffentlich geförderte }\end{array}\end{array}$ & $13,2 \%$ & $18,6 \%$ & $17,2 \%$ & $0 \%$ \\
$\begin{array}{l}\text { WB-Einrichtungen } \\
\text { Freie Träger der WB }\end{array}$ & $11,8 \%$ & $27,1 \%$ & $41,7 \%$ & $2,9 \%$ \\
$\begin{array}{l}\text { Kultureinrichtungen } \\
\text { Schulen }\end{array}$ & $18,6 \%$ & $7,8 \%$ & $7,8 \%$ & $1,5 \%$ \\
Sonstige & $25,5 \%$ & $11,3 \%$ & $4,4 \%$ & $0,5 \%$ \\
\hline
\end{tabular}

Tab. 6: Kooperationspartner und Ebenen der Kooperation $(\mathrm{N}=204)$. 
Besonders die gemeinsame Nutzung von Ressourcen mit Schulen (60,8\%) und der Austausch von Kursleitenden mit anderen öffentlich geförderten Weiterbildungseinrichtungen (41,7\%) ragen hier heraus. Die große Anzahl an Nennungen der Kooperation mit der Schule weist darauf hin, dass viele Einrichtungen auf der einen Seite nicht über ausreichende eigene Infrastruktur verfügen, um Angebote mit Neuen Medien zu realisieren, auf der anderen Seite durch diese Kooperation den finanziellen Aufwand reduzieren können. Diese Kooperation erscheint vor allem auch sinnvoll, weil die Nutzungszeiten von Schule und Weiterbildungseinrichtungen sich gut ergänzen.

Betrachtet man die Gesamtstruktur der Kooperationsbeziehungen, wird deutlich, wie vielschichtig sich das Kooperationsnetz der Einrichtungen darstellt. Insgesamt lässt sich feststellen, dass die Einrichtungen im Bereich Neue Medien über eine nennenswerte Kooperationskultur verfügen.

Doch noch mehr als die Kooperation wird im Bereich der Neuen Medien die Konkurrenz gesehen. Auf die Frage „Sehen Sie für Ihre Einrichtung in diesem Bereich eine Konkurrenz durch andere Anbieter?“ antworten 81,2\% ( $=372)$ mit „Ja“. In der folgenden Tabelle ist aufgezeigt, durch welche Anbieter diese gesehen wird (die Optionen waren vorgegeben, Mehrfachnennungen möglich).

\begin{tabular}{ll}
\hline Private kommerzielle Bildungsanbieter & $89,9 \%$ \\
Freie Träger der Weiterbildung & $62,4 \%$ \\
Öffentlich geförderte WB-Einrichtungen & $61,1 \%$ \\
Kultureinrichtungen (z.B. Bibliotheken, Museen) & $12,7 \%$ \\
Sonstige & $4,2 \%$ \\
\hline
\end{tabular}

Tab. 7: Konkurrenz im Bereich Neue Medien $(\mathrm{N}=306)$.

Es wird deutlich, dass die privaten kommerziellen Bildungsanbieter als Hauptkonkurrenten gesehen werden.

Betrachtet man die Dimensionen „Kooperation“ und „Konkurrenz“ in der Zusammenschau wird die Ambivalenz deutlich. Auf der einen Seite gibt es eine nennenswerte Kooperationskultur, auf der anderen Seite werden die potenziellen Kooperationspartner auch als Konkurrenten gesehen. An dieser Stelle wäre es interessant zu untersuchen, ob durch verstärkte Kooperationen Synergieeffekte z.B. im Hinblick auf neue Zielgruppen erzielt werden können.

\section{$4.5 \quad$ Neue Medien und Organisationsentwicklung}

Eine der zentralen Fragestellungen der vorliegenden Untersuchung fokussiert die Bedeutung der Neuen Medien für die Organisationsentwicklung der Einrichtungen. Für 59,7\% spielen bzw. spielten die Neuen Medien eine zentrale Rolle in diesen Prozessen. Ein Blick darauf, wie stark nach Einschätzung der Einrichtungen sich einzelne Bereiche in der Einrichtung in den letzten fünf Jahren durch die Neuen Medien verändert haben, gibt einen Überblick über 
die Struktur von Veränderungen ${ }^{13}$. Ordnet man die Bereiche nach der Höhe der Mittelwerte ergibt sich folgendes Bild:

\begin{tabular}{ll}
\hline Arbeitsabläufe in der Verwaltung & 4,63 (Standardabw.: 1,$30 ; \mathrm{N}=374$ ) \\
Angebot & 4,30 (Standardabw.: 1,13; $\mathrm{N}=372$ ) \\
Institution insgesamt & 3,83 (Standardabw.: 1,25; $\mathrm{N}=366$ ) \\
Adressaten- und Teilnehmendenstruktur & 3,45 (Standardabw.: 1,24; $\mathrm{N}=360$ ) \\
Externe Zusammenarbeit & 2,81 (Standardabw.: 1,32; $\mathrm{N}=366$ ) \\
Zusammenarbeit zwischen Planenden und Kursleitenden & 2,80 (Standardabw.: 1,26; $\mathrm{N}=370$ ) \\
Veranstaltungsformen & 2,75 (Standardabw.: 1,28; $\mathrm{N}=366$ ) \\
Interne Zusammenarbeit & 2,59 (Standardabw.: 1,38, $\mathrm{N}=356$ ) \\
\hline
\end{tabular}

Tab. 8: Veränderungen durch Neue Medien nach eingeschätzter Stärke.

Betrachtet man die Einschätzungen bezüglich zukünftiger Veränderungsbedarfe in unterschiedlichen Bereichen der Einrichtungen ${ }^{14}$, dann fällt auf, dass auch dort vor allem für die interne Organisationsstruktur der geringste Veränderungsbedarf gesehen wird.

\begin{tabular}{ll}
\hline Angebotsentwicklung & 4,26 (Standardabw. 1,08; $\mathrm{N}=370$ ) \\
Personalentwicklung/Fortbildung & 4,10 (Standardabw. 1,23; $\mathrm{N}=365$ ) \\
Entwicklung veränderter didaktisch-methodischer Konzepte & 3,94 (Standardabw. 1,25; $\mathrm{N}=365$ ) \\
Zielgruppenorientierung & 3,85 (Standardabw. 1,23; $\mathrm{N}=366$ ) \\
Organisationsentwicklung & 3,81 (Standardabw. 1,27; $\mathrm{N}=366$ ) \\
Externe Kooperation & 3,76 (Standardabw. 1,35; $\mathrm{N}=365$ ) \\
Gestaltung von neuen Lernarrangements & 3,58 (Standardabw. 1,48; $\mathrm{N}=366$ ) \\
Interne Kommunikation & 3,57 (Standardabw. 1,51; $\mathrm{N}=368$ ) \\
Fachbereichsgliederung & 2,47 (Standardabw. 1,25; $\mathrm{N}=354$ ) \\
\hline
\end{tabular}

Tab. 9: Veränderungsbedarfe im Hinblick auf Neue Medien.

Zwar wird der Veränderungsbedarf im Bereich „Personalentwicklung/Fortbildung“ als sehr hoch eingeschätzt, was in Anbetracht der Konkurrenzsituation, in der sich viele Einrichtungen sehen, auch von erheblicher Bedeutung ist, doch die „Interne Kommunikation“ und die traditionelle „Fachbereichsgliederung“ werden im Hinblick auf die Neuen Medien als nicht besonders veränderungsbedürftig angesehen. Viele Einrichtungen sind anscheinend in der Tendenz mit den internen Organisationsstrukturen zufrieden.

Die Ergebnisse legen nahe zu vermuten, dass das große Interesse an Organisationsentwicklung vor allem auf das Interesse an Verbesserungen im

13 Die acht Bereiche waren im Fragebogen vorgegeben. Die Stärke der Veränderung konnte anhand einer Skala von 1 bis 6 eingeschätzt werden (Skalenwert „1“ = „Überhaupt nicht“; Skalenwert „,“ = „Ganz stark“).

14 Auch hier waren die ereiche im Fragebogen vorgegeben. Die Größe des Veränderungsbedarfs konnte anhand einer Skala von 1 bis 6 eingeschätzt werden (Skalenwert „1“ = „Überhaupt keinen“; Skalenwert „6“ = „Besonders großen“). 
Bereich der Außenbeziehungen zurückzuführen ist. Dies wird bestätigt durch die Antworten auf die Frage, welche Veränderungen durch den Einsatz Neuer Medien in der Öffentlichkeitsarbeit der Einrichtungen festzustellen sind ${ }^{15}$. 87,8\% $(\mathrm{N}=376)$ sehen hier den „Imagegewinn“, 84,0\% die „Erreichung neuer Zielgruppen“ und 58,2\% „,effektivere Arbeitsabläufe“ als Effekte. „Imagegewinn“ als Effekt ist sicher eine wichtige Motivation für den Einsatz Neuer Medien. Es könnte sich allerdings perspektivisch als Problem erweisen, wenn nicht gleichzeitig in der Binnenstruktur der Einrichtung Veränderungsprozesse - über die Veränderungen im Verwaltungsbereich hinaus - vorgenommen werden. Hierin liegt für die Zukunft ein wichtiges Feld für die Organisationsentwicklung der Einrichtungen, wenn sie sich nicht nur an Verbesserungen der Außendarstellung orientieren soll, sondern wenn die Neuen Medien auch als zentrales Instrument der internen Weiterentwicklung genutzt werden sollen.

\subsection{Situation in ausgewählten Einrichtungen}

Auf die detaillierte Darstellung der Analyse der Interviews muss an dieser Stelle verzichtet werden. Es geht hier vor allem darum, einige Aspekte aus den Interviews festzuhalten, die mit Bezug auf die anderen Ergebnisse von besonderer Relevanz sind.

Betrachtet man die Situation in den ausgewählten Einrichtungen insgesamt, wird deutlich, dass in allen Einrichtungen bei der Leitung eine große Aufgeschlossenheit gegenüber den Neuen Medien festzustellen ist, ohne dass sie selbst Experten in diesem Bereich sind. Bei den EDV-Verantwortlichen fällt der engagiert-pragmatische Umgang mit den Neuen Medien auf, der sich dadurch auszeichnet, dass sie den Einsatz Neuer Medien nicht per se positiv beurteilen, sondern Möglichkeiten eines sinnvollen Einsatzes abhängig vom Verwendungskontext machen.

Von beiden Expertengruppen gehen Impulse für die Entwicklung im Bereich Neue Medien in den jeweiligen Einrichtungen aus, und die EDV-Verantwortlichen werden von der Leitung unterstützt. Außerdem wurde in den Experteninterviews deutlich, dass das Verhältnis zwischen Leitung und EDV-Verantwortlichen nicht nur in den kleinen Einrichtungen, die über wenig Personal verfügen, sondern auch in den größeren Einrichtungen als sehr gut bezeichnet werden kann. In keiner der Einrichtungen wurde eine explizite Kritik am jeweils anderen Akteur geäußert.

Offene Kommunikationsstrukturen, teamorientierte Entscheidungsprozesse, Interesse an zielgerichteten Veränderungen und eine jeweils sehr spezifische Organisationskultur kennzeichnen den Zugang der Einrichtungen zu den Neuen Medien. Darüber hinaus können folgende Faktoren in Bezug auf die Neuen Medien in den ausgewählten Einrichtungen festgehalten werden:

15 Die Bereiche waren im Fragebogen vorgegeben. 
- Offenheit der Leitung,

- engagierter Pragmatismus bei den EDV-Verantwortlichen,

- Unterstützung des EDV-Verantwortlichen durch die Leitung,

- Impulse für die Entwicklung sowohl von der Leitung als auch von den EDV-Verantwortlichen.

Die strategische Ausrichtung der Einrichtungen stellt sich auf der einen Seite sehr unterschiedlich dar, da sich die Einrichtungen strukturell sehr unterscheiden, auf der anderen Seite lassen sich doch ein paar Übereinstimmungen feststellen. Dass die Angst davor, den Anschluss zu verlieren, ein Motor für die Entwicklungen im Bereich der Neuen Medien ist, machen die folgenden Aussagen deutlich:

„Wenn wir da nicht schritt gehalten hätten mit den anforderungen sozusagen des arbeitsmarktes (...) und wenn wir das nicht im programm platziert hätten, in unser angebot und die technik sozusagen hätten links liegen lassen, um es mal so zu sagen, dann wären wir eine arme volkshochschule geworden" (A-Leitung: 65).

„Wir haben gesagt: das müssen wir machen, wenn wir das nicht machen, sind wir weg vom fenster" (C-Leitung: 49).

Dass sich die strategische Ausrichtung auch stark an Kooperationsoptionen orientiert, zeigt sich bei Einrichtung E:

„Das war für uns irgendwann auch mal der punkt zu sagen o.k. wir machen das, wir ziehen das durch oder wir klinken uns aus. Wir haben uns für das erstere entschlossen, bzw. der entschluss war an sich verhältnismäßig einfach zu fällen oder umzusetzen, weil wir zu einem sehr frühen zeitpunkt hier innerhalb der verwaltung der stadt uns bemüht haben, entsprechende angebote zu machen in bezug auf die mitarbeit in der schulung der verwaltung" (E-Leitung: 35).

Von vornherein stärker den Markt im Blick hatte die Einrichtung D:

„Dass das immer mehr nachgefragt worden ist und dass wir in diesen dingen einen markt gewittert haben, die erwachsenenbildung einfach attraktiver, einfach dem konsumenten, der da war, den man nicht suchen musste, sondern der nachfragte, dem adäquat etwas zu bieten“" (D-Leitung: 27).

Eine Politik der kleinen Schritte fährt die Einrichtung B:

„Wir haben eigentlich bei uns in der institution immer die politik gefahren, dass wir gesagt haben, wir gehen mit kleinen schritten voran, und wir versuchen, immer aus unseren erfahrungen heraus, dann erst den nächsten schritt zu tun, also wirklich, wie man so schön sagt: induktiv vorzugehen und nicht deduktiv irgendein konzept oben draufzusetzen und dann zu schauen, funktioniert es oder wollen die leute das überhaupt“ (B-Leitung: 23).

Die ausgewählten Einrichtungen haben sich, mit Ausnahme von Einrichtung A, die auch durch landesgesetzliche Rahmenbedingungen bedingt ihr Dienstleistungsangebot kaum ausbauen kann, ihre speziellen Nischen bzw. ihre besonderen Profile unabhängig vom traditionellen Kursangebot geschaf- 
fen. So haben sich zum Beispiel die Einrichtungen B und E vor allem mit den Fortbildungen für die Stadt- und Kreisverwaltung profiliert und sich hier ein starkes Standbein erarbeitet. Die Einrichtung C arbeitet im Dienstleistungsbereich mit einer überregional agierenden Organisation zusammen und deckt dort einen großen Teil des Schulungsbedarfs im Bereich Neue Medien ab, außerdem arbeitet sie im Schulungsbereich für Firmen und Stadtverwaltungen. Die Schulung von Firmenpersonal und Maßnahmen für das Arbeitsamt sind Schwerpunkte der Einrichtung D, die in ihrer Region hier eine herausragende Stellung einnimmt. Es kann an dieser Stelle festgehalten werden, dass die Entwicklung eines eigenständigen Dienstleistungsprofils ein Faktor für die aktive Entwicklung im Bereich Neue Medien in den Einrichtungen darstellt bzw. hier ein Wechselspiel festzustellen ist.

Bei allen Einrichtungen fällt auf, dass sie mit neuen Angebotsformen experimentieren bzw. experimentiert haben. Dabei geht es in der Hauptsache zum einen um Selbstlernzentren, zum anderen um internetbasierte Angebote.

Auch wenn die strategische Ausrichtung der ausgewählten Einrichtungen bzgl. der Neuen Medien nicht als besonders ungewöhnlich bezeichnet werden kann, zeigen sich doch zwei Bereiche, die besonders hervorgehoben werden müssen:

- das spezifische Dienstleistungsprofil und

- die Bereitschaft zum Experiment.

Es bleibt festzuhalten, dass diese beiden Bereiche jeweils mehr oder weniger kennzeichnend für alle ausgewählten Einrichtungen sind. Nimmt man die unterschiedliche strukturellen Verfassungen der Einrichtungen, treten diese beiden Ebenen noch stärker als übergreifende Faktoren für im Bereich Neue Medien innovativ agierende Einrichtungen zutage.

\section{Anforderungen an eine medienorientierte Organisationsentwicklung}

Bei der Gesamtschau der Ergebnisse muss nochmals auf die große Heterogenität der Einrichtungen hingewiesen werden. Das Spektrum reicht von der ehrenamtlich geleiteten Volkshochschule in der ländlichen Region ohne hauptberufliche pädagogische Mitarbeiter/innen bis hin zur großstädtischen Volkshochschule mit über hundert hauptberuflichen pädagogischen Mitarbeiter/innen.

Luhmann weist mit dem Bezug auf die analytische Kategorie der Organisationskultur darauf hin, dass die jeweilige Geschichte einer Organisation das System individualisiert (vgl. 2000, S. 248) und damit die Grenzen der Generalisierbarkeit von empirischen Befunden deutlich wird. Dies verweist darauf, dass bei der Analyse von Organisationen der Blick auf die einzelne 
Organisation unter empirischen Gesichtspunkten von großer Bedeutung ist. Deshalb erfolgte in der vorliegenden Untersuchung auch der Blick auf einzelne Organisationen. Trotzdem lassen sich einige übergreifende Aspekte formulieren, die für das Verhältnis von Neuen Medien und Organisation in Weiterbildungseinrichtungen symptomatisch sind.

Die hier vorgestellten Ergebnisse ergeben ein ambivalentes Bild der Beziehung von Neuen Medien und den Einrichtungen. Die große Aufgeschlossenheit und die gute technische Infrastruktur weisen auf der einen Seite in der Tendenz auf Innovationsbereitschaft bzw. auf die Bereitschaft zur Anpassung auf die von außen an die Einrichtungen herangetragenen Bedarfe hin, die traditionellen Angebotsformen und das Festhalten an internen Organisationsstrukturen auf der anderen Seite auf eine fehlende grundsätzliche Veränderungsbereitschaft, die mit unterschiedlichen Faktoren zusammenhängen kann, wie z.B.:

- fehlende finanzielle und personelle Ressourcen

- Skepsis gegenüber den Neuen Medien

- fehlende Innovationsbereitschaft bei der Leitung.

Vor allem der letzte Punkt ist einer der zentralen Faktoren, die die Weiterentwicklung von Einrichtungen im Bezug auf die Neuen Medien beeinflussen. Dies haben die Ergebnisse der Auswertung von Experteninterviews gezeigt.

Ingesamt bietet die vorgestellte Untersuchung eine erste Grundlage für die Analyse der Frage, wie sich die Neuen Medien auf die Organisation von Weiterbildungseinrichtungen auswirken. In diesem Beitrag konnte es nur darum gehen, einige ausgewählte Aspekte des Einsatzes Neuer Medien in Volkshochschulen anhand der empirischen Untersuchung darzustellen und auf einige Problemfelder hinzuweisen, die es in Zukunft sowohl durch weitere Untersuchungen als auch durch die Entwicklung von Umsetzungskonzepten zu bearbeiten gilt. Folgende Fragestellungen lassen sich hier exemplarisch festhalten:

- Wie kann eine professionsadäquate Betreuung der technischen Infrastruktur gewährleistet werden?

- Welche internen Organisationsstrukturen zur Weiterentwicklung im Bereich Neue Medien werden benötigt?

- Wie können die Neuen Medien stärker für die Organisationsentwicklung genutzt werden?

- Wie könnte eine medienorientierte Organisationsentwicklung gestaltet sein?

- Wie lässt sich ein gezieltes Innovationsmanagement in den Einrichtungen realisieren?

Diese und weitere Fragen, die sich aus der Analyse der Situation in den Volkshochschulen ergeben, betreffen nicht nur die Volkshochschulen, sondern sind für alle Weiterbildungseinrichtungen von Relevanz, die in lokalen 
und regionalen Kontexten agieren. Für die Zukunft wäre es von großem Interesse, auch andere Trägerbereiche im Hinblick auf das Thema „Neue Medien und Organisation“ zu untersuchen, da es bislang in der Breite an einer empirischen Grundlage zur Bearbeitung der genannten Fragestellungen fehlt. Mit der hier vorgestellten Untersuchung ist ein Anfang gemacht.

Betrachtet man vor allem die Ergebnisse der Experteninterviews, lassen sich einige Faktoren herausarbeiten, die sich fördernd auf eine medienorientierte Organisationsentwicklung auswirken. In der Zusammenfassung der Ergebnisse lassen sich einige Eckpunkte aufzeigen, die allgemein für die Entwicklung von medienbezogenen Angeboten von Relevanz sein können.

Die Entwicklung neuer Angebote muss sich zwar immer an den jeweiligen strukturellen Rahmenbedingungen der Einrichtungen orientieren. Doch hat sich in der Untersuchung gezeigt, dass auch kleinere Einrichtungen mit einer geringen personellen Ausstattung in der Lage sind, innovative Angebote auch im Bereich Neue Medien zu gestalten. Die finanziellen und personellen Rahmenbedingungen sind zwar ein wichtiger Faktor bei einer medienorientierten Entwicklung von Weiterbildungseinrichtungen, doch nicht der allein entscheidende. Vielmehr ist es das Zusammenspiel unterschiedlicher Aspekte, die zu berücksichtigen sind, wenn man eine medienorientierte Entwicklung von Weiterbildungseinrichtungen vorantreiben will. Die Organisationskultur ist sicher einer der entscheidenden Faktoren. Die Organisationskultur lässt sich aus verschiedenen Perspektiven beleuchten. Im Folgenden werden einige zentrale Dimensionen aufgezeigt.

\section{Restrukturierung und Organisationsentwicklung}

Der Einsatz Neuer Medien führt zu Veränderungen in der Organisation von Weiterbildungseinrichtungen. Zunächst ist davon vor allem der Verwaltungsbereich betroffen, doch hat diese Entwicklung auch Auswirkungen auf den pädagogischen Bereich. Dieses zu erkennen und produktiv in neue Organisationskonzepte umzusetzen ist eine der zentralen Herausforderungen für die Weiterbildungseinrichtungen. Es gilt diese gezielt in Angriff zu nehmen, wie die Untersuchung einzelner Einrichtungen gezeigt hat. Dabei gibt es keine Patentrezepte, sondern jede Einrichtung muss entsprechend ihrer Organisationskultur die Neuen Medien in die Weiterentwicklung der Einrichtung integrieren.

\section{Offenheit und Innovation}

Offenheit und Innovationsbereitschaft bei der Leitung von Weiterbildungseinrichtungen sind wichtige Faktoren, wenn es darum geht, medienbezogene Angebote in Angriff zu nehmen. In der Untersuchung hat sich gezeigt, dass dort, wo die Initiative zu neuen Angeboten von der Leitung ausgeht bzw. Initiativen der Mitarbeitenden von der Leitung unterstützt werden, die Entwicklung von neuen Angeboten stärker vorangetrieben wird. 
Dazu gehört auch, dass das Experimentieren mit neuen Angebotsformen zu einem wichtigen Element der Einrichtungspolitik wird. Dabei sollte vor allem auch darauf geachtet werden, welche Bedarfe sich von Seiten der Adressat/innen ergeben und welche Anregungen die Kursleitenden, die oft auch aus Arbeitszusammenhängen der Wirtschaft kommen, geben. Die Sensibilität für die Veränderungen des Umfeldes, z.B. neuer Qualifikationsbedarfe, ist ein entscheidender Faktor dabei, aktuelle Trends zu erkennen und für die Einrichtung nutzbar zu machen.

Um ein gezieltes Innovationsmanagement in Sachen Neue Medien zu betreiben, gilt es, in der Institution organisatorische Strukturen zu entwickeln, wie z.B. Projektgruppen, die programmbereichsübergreifend Ideen, Anregungen und bereits in anderen Einrichtungen gemachte Erfahrungen bündeln und daraus ein strategisches Konzept für die eigene Einrichtung entwickeln.

\section{Experiment und Erprobung}

Auch wenn neue medienbasierte Angebotsformen auf weiterbildungspolitischer Ebene gefordert werden und Adressat/innen von Weiterbildungsangeboten den Bedarf nach Online-Angeboten formulieren, ist doch zum Beispiel der Bereich des Telelernens in Weiterbildungskontexten in Deutschland bislang nur unzureichend entwickelt, so dass sich nur bedingt auf gut aufbereitete Praxiserfahrungen zurückgreifen lässt. Deshalb ist jedes Projekt der Angebotsentwicklung von Telelernen nach wie vor ein Pilotprojekt, das Experimentcharakter hat. Darauf sollten sich Weiterbildungsinstitutionen einrichten, wenn sie solche Angebote planen.

Das bedeutet auch, dass nur bedingt auf fertige Konzepte für lokal bzw. regional agierende Weiterbildungseinrichtungen zurückgegriffen werden kann - wenn es hier auch erste Ansätze z.B. im Kontext der Entwicklung des Angebots „Virtuelle Volkshochschule“ (www.vhs-virtuell.de) gibt.

\section{Evaluation und Bewertung}

Neue Angebotsformen - wie zum Beispiel Online-Angebote - müssen während und nach der Experimentierphase, die auch zur Revision des Ansatzes genutzt werden soll, auf ihre Angemessenheit im Hinblick auf Marktumfeld, den Bedarf bei den Adressant/innen sowie die personellen und finanziellen Ressourcen evaluiert werden. Dazu sollte die jeweilige Projektgruppe in den Einrichtungen die Realisierung des Experiments begleiten und externe Berater/innen hinzugezogen werden.

Dadurch kann gewährleistet werden, dass die Angebote im Hinblick auf sowohl die Kapazitäten und Entwicklungsperspektiven der Weiterbildungseinrichtung (intern) als auch den Vergleich mit anderen Einrichtungen unter der Perspektive des Benchmarking (extern) bewertet werden können.

Wichtig bei der Bewertung der Experimentierphase sind nicht nur die harten wirtschaftlichen Fakten wie z.B. Ausgaben/Einnahmen, sondern auch 
die Auseinandersetzung mit der Frage, wie sich z.B. das Image der Einrichtungen durch Online-Angebote verändert. In der Studie hat sich gezeigt, dass der Großteil der Einrichtungen einen Imagegewinn durch den Einsatz der Neuen Medien für ihre Einrichtung konstatiert. Darüber hinaus wurde deutlich, dass Einrichtungen, die besonders offensiv im Bereich der Neuen Medien agieren, unter anderem von Firmen und Behörden als auf diesem Feld kompetente Institution wahrgenommen werden und sich dadurch neue Geschäftsfelder im Schulungsbereich eröffnen.

\section{Vernetzung und Kooperation}

Medienbezogene Angebote sind oft kostenintensiv. Um trotzdem in diesem Bereich aktiv sein zu können und dadurch auch das Image der Einrichtung zu verbessern, bieten sich Kooperationen mit anderen Weiterbildungseinrichtungen an. Dies kann auf der einen Seite dadurch realisiert werden, dass sich Einrichtungen aus dem gleichen Trägerspektrum zusammentun (z.B. Volkshochschulen im regionalen Kontext) oder auf der anderen Seite dadurch, dass sich übergreifend Weiterbildungseinrichtungen lokal bzw. regional vernetzen und ein gemeinsames Medien-Portal entwickeln und dieses auch gemeinsam vermarkten. Auch wenn besonders die zweite Lösung unter der Konkurrenzperspektive eher dysfunktional erscheinen mag, ist doch darauf hinzuweisen, dass sich durch eine solche Kooperation neue Zielgruppen erreichen lassen, Ressourcen gebündelt werden und der Imageeffekt allen Einrichtungen zugute kommt. Vor allem im Hinblick auf die Verhandlungen mit politischen Entscheidungsträgern (z.B. über Projektfinanzierungen) erscheint ein solcher Weg lohnenswert, dies besonders auch, wenn man die derzeitige Diskussion über „Lernende Regionen“ betrachtet.

\section{Professionalisierung und Interdisziplinarität}

Bezogen auf den Einsatz Neuer Medien wurden in der Untersuchung unter anderem zwei Problembereiche deutlich: Zum einen fehlt es bislang in der Breite an einer umfassenden Professionalisierung der pädagogisch Verantwortlichen im Bereich Neue Medien, zum anderen konzentrieren sich die Angebote zu dem Themenfeld Neue Medien vor allem auf den Bereich „Arbeit und Beruf“.

Da die Entwicklung von Medienangeboten für eine Weiterbildungseinrichtung eine Grundsatzentscheidung darstellt, die Auswirkungen auf die gesamte Organisation hat, entsteht hier nicht nur ein Fortbildungsbedarf für die direkt damit Beschäftigten, sondern für alle Mitarbeiter/innen der Einrichtung, auch im Bereich der Verwaltung. Eine medienorientierte Organisationsentwicklung muss einhergehen mit einer medienorientierten Personalentwicklung.

Da die Entwicklung der technischen Infrastruktur unabhängig von Inhalten realisiert wird und somit potenziell für alle Inhaltsbereiche zur Verfügung gestellt werden kann, sollte in den Weiterbildungseinrichtungen geklärt 
werden, welche Inhaltsbereiche die technische Infrastruktur nutzen können und sollen. Das bedeutet, dass in medienbezogenen Projektgruppen nicht nur ein thematischer Bereich wie berufliche Bildung oder EDV-Schulung vertreten ist, sondern möglichst das gesamte Spektrum der Einrichtung. Nur so können neue Angebotsformen interdisziplinär entwickelt werden, und dies ist langfristig eine der Voraussetzungen für den Erfolg. Denn eine Einrichtung wird sich erst dann als Online-Anbieter etablieren können, wenn sie ein möglichst breites Spektrum abdeckt.

\section{Zusammenfassende Betrachtungen}

Im Rahmen dieses Beitrages konnten nur einige Aspekte des Verhältnisses von Neuen Medien und Organisation präsentiert werden. Die Darstellung des forschungsmethodischen Kontextes sollte den empirischen Ansatz dieser Untersuchung deutlich machen. Die Bezüge der Untersuchung auf die Luhmannsche Systemtheorie konnten hier nur angedeutet werden, doch zeigt sich, dass sein Konzept der „Organisationskultur“, das darauf verweist, dass sich jede Organisation durch spezifische Kommunikations-, Entscheidungs- und Reformstrukturen auszeichnet, äußerst hilfreich für die Untersuchung von Weiterbildungseinrichtungen ist. Denn erst durch die Untersuchung einzelner Einrichtungen mit ihren jeweils spezifischen Strukturen lassen sich Konzepte des Umgangs von Organisationen mit den Neuen Medien herausarbeiten.

Auch wenn es Ansätze einer pädagogischen Organisationstheorie gibt (vgl. z.B. Schäffter 1998), bleibt doch festzustellen, dass es bezogen auf die Auseinandersetzung mit den gravierenden Veränderungen durch die Neuen Medien an einer ausgearbeiteten Organisationstheorie fehlt. Dies erweist sich vor allem vor dem Hintergrund als Problem, da die organisatorische Rahmung grundlegend für das pädagogische Handeln ist. Deshalb könnte eine intensivere Auseinandersetzung mit Fragen der institutionellen Organisation von Bildungseinrichtungen im Verhältnis zur Medienentwicklung für die Zukunft auch ein wichtiges Feld für die medienpädagogische Forschung darstellen. Ich hoffe, dass mit der vorliegenden Untersuchung ein kleiner Beitrag dazu geleistet werden konnte, die Sensibilität gegenüber diesen Fragestellungen zu erhöhen.

\section{Literatur}

Backhaus, K./Erichson, B./Plinke, W./Weiber, R.: Multivariate Analysemethoden. Eine anwendungsorientierte Einführung. 9., überarb. und erw. Aufl.. Berlin u.a. 2000 [1980].

Beck, U.: Risikogesellschaft. Frankfurt a.M. 1986.

Gross, P.: Die Multioptionsgesellschaft. Frankfurt a.M. 1994.

Lamnek, S.: Qualitative Sozialforschung. Band 2: Methoden und Techniken. 2., überarb. Aufl.. Weinheim 1993.

Luhmann, N.: Organisation und Entscheidung. Opladen/Wiesbaden 2000.

Meiser, T./Humburg, S.: Klassifikationsverfahren. Erdfelder, E. u.a. (Hrsg.): Handbuch Quantitative Methoden. Weinheim 1996, S. 279-290. 
Meuser, M./Nagel, U.: Das ExpertInneninterview - Wissenssoziologische Voraussetzungen und methodische Durchführung. In: Friebertshäuser, B./Prengel, A. (Hrsg.): Handbuch Qualitative Forschungsmethoden in der Erziehungswissenschaft. Weinheim/München 1997, S. 481-491.

Pehl, K./Reitz, G.: Volkshochschulstatistik. Arbeitsjahr 1999. Hrsg. vom Deutschen Institut für Erwachsenenbildung. Bielefeld 2000.

Rogers, E. M.: Diffusion of Innovations. 4. Aufl.. New York: The Free Press 1995 (1962).

Rudolf, K.: Telelernen in der Volkshochschule. Hrsg. vom Deutschen Volkshochschul-Verband Netzwerk Beruf und Weiterbildung. Bonn/Frankfurt a.M. 2001.

Schäffter, O.: Organisation. In: Arnold, R./Nolda, S./Nuissl, E. (Hrsg.): Wörterbuch Erwachsenenpädagogik. Bad Heilbrunn/Obb. 2001, S. 243-246.

Schäffter, O.: Weiterbildung in der Transformationsgesellschaft. Zur Grundlegung einer Theorie der Institutionalisierung. Berlin 1998.

Schnell, R./Hill, P.B./Esser, E.: Methoden der empirischen Sozialforschung. München/Wien 1995.

Schütz, A.: Der gut informierte Bürger. Ein Versuch über die soziale Verteilung des Wissens. In: Schütz, A.: Gesammelte Aufsätze. Band 2: Studien zur soziologischen Theorie. Den Haag 1972, S. 85-101.

Schulze, G.: Die Erlebnisgesellschaft. Kultursoziologie der Gegenwart. Frankfurt a.M./New York 1993.

Schwarzer, B./Krcmar, H.: Wirtschaftsinformatik. Grundzüge der betrieblichen Datenverarbeitung. Reihe: Praxisnahes Wirtschaftsstudium. 2., überarb. und erw. Aufl.. Stuttgart 1999.

Stehr, N.: Die Zerbrechlichkeit moderner Gesellschaften. Die Stagnation der Macht und die Chancen des Individuums. Weilerswist 2000.

Voß, W.: Praktische Statistik mit SPSS. 2., aktual. Aufl.. München/Wien 2000.

Wagemann, M./Stang, R.: Multimedia in der Erwachsenenbildung. Ergebnisse einer Befragung. In: Stang, R./Apel, H./Hagedorn, F. (Hrsg.): Pädagogische Innovation mit Multimedia. Band 3: Konzepte, Analysen, Perspektiven. Frankfurt a.M. 1999, S. 113-125. 